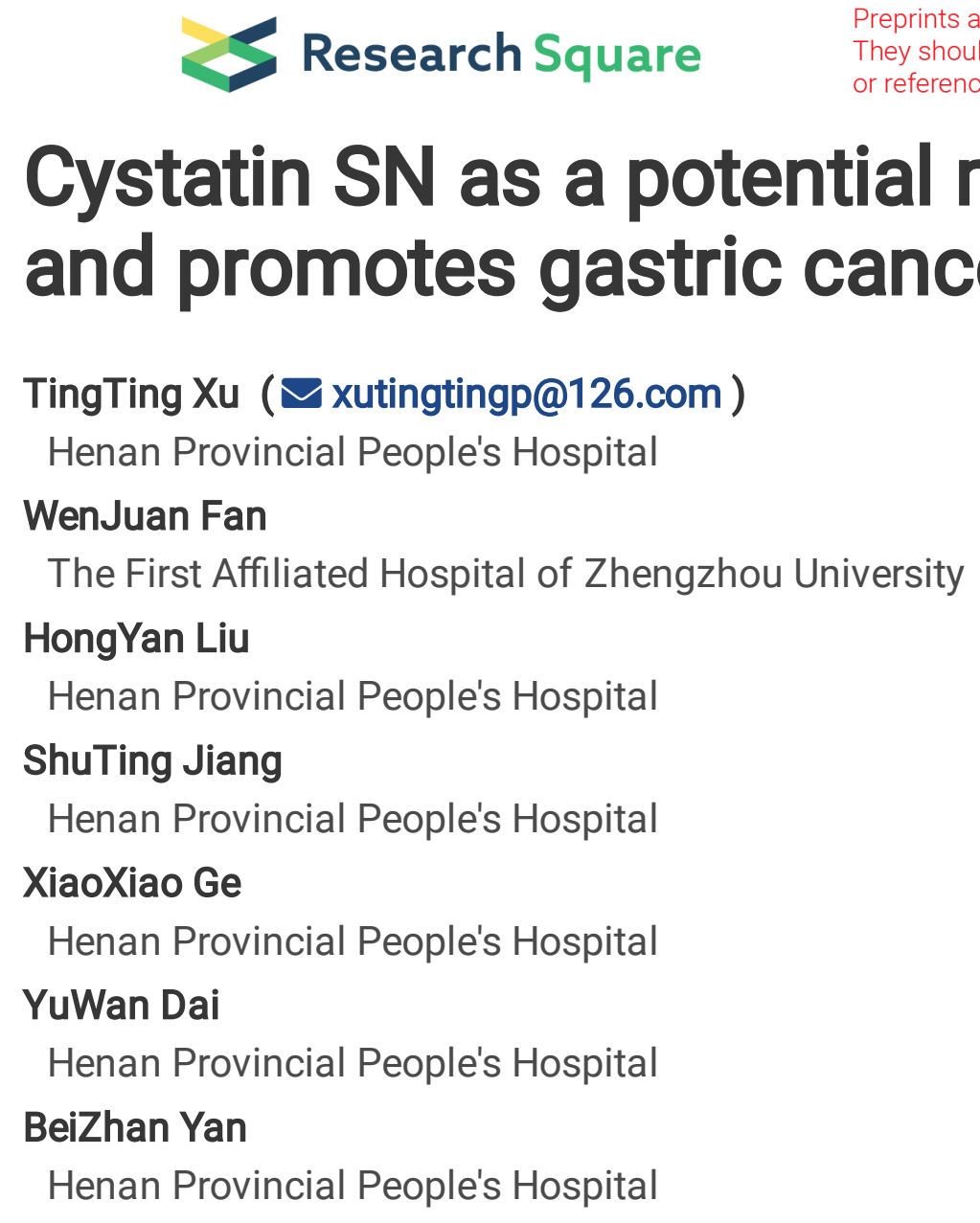

\author{
TingTing Xu ( $\nabla$ xutingtingp@126.com ) \\ Henan Provincial People's Hospital \\ WenJuan Fan \\ The First Affiliated Hospital of Zhengzhou University \\ HongYan Liu \\ Henan Provincial People's Hospital \\ ShuTing Jiang \\ Henan Provincial People's Hospital \\ XiaoXiao Ge \\ Henan Provincial People's Hospital \\ YuWan Dai \\ Henan Provincial People's Hospital \\ BeiZhan Yan \\ Henan Provincial People's Hospital
}

\title{
Cystatin SN as a potential marker for gastric cancer and promotes gastric cancer progression
}

\section{Research Article}

Keywords: Gastric cancer, CST1, Prognosis, Genetic alterations

Posted Date: March 2nd, 2022

DOI: https://doi.org/10.21203/rs.3.rs-1345245/v1

License: (c) (1) This work is licensed under a Creative Commons Attribution 4.0 International License.

Read Full License 


\section{Abstract}

\section{Background}

Cystatin SN (cystatin 1, CST1) regulates the progression of many cancers and serves as a diagnostic marker; however, its significance in gastric cancer has not been fully elucidated.

\section{Methods}

We first analyzed the expression levels of CST1 in gastric cancer dataset in oncomine and UALCAN databases. We next analyzed the relationship between CST1 and prognosis of gastric cancer patients in KM Plotter database and downloaded TCGA GC data to verify this result. We further divided GC patients into two groups according to the level of CST1 expression for GSEA enrichment analysis. Finally, we validated the role of CST1 in gastric cancer by in vitro functional assays.

\section{Results}

We found that CST1 expression levels in gastric cancer tissues were significantly higher than those in normal control tissues and patients with high CST1 expression had a poorer prognosis. GSEA enrichment analysis showed that the main KEGG signaling pathways enriched in the CST1 high expression group were Coagulation, Epithelial-Mesenchymal-Transition, Complement, Apoptosis, Glycolysis. In vitro functional assay confirmed that inhibition of CST1 significantly inhibited the proliferation, colonyformation,migration and invasion ability of gastric cancer cells.

\section{Conclusions}

Our study shows that the expression level of CST1 correlates with the development of gastric cancer, and CST1 may be used as a diagnostic marker for poor prognosis of gastric cancer.

\section{Background}

Global cancer statistics analysis in 2018 showed that gastric cancer has more than 1 million new cases and about 783,000 deaths per year, making it the fifth most prevalent malignancy and the third most deadly malignancy worldwide $(1,2)$. Gastric cancer is insidious and difficult to diagnose at an early stage, and some patients are already at an advanced stage when diagnosed, losing the opportunity for radical surgery, while even the recurrence rate is high after surgical resection, resulting in a poor overall prognosis for gastric cancer patients. Finding diagnostic and therapeutic related tumor markers with high specificity and sensitivity is of great significance to improve the survival rate and prolong the survival period of gastric cancer patients.

CST1 is a cysteine protease inhibitor that belongs to the cysteine superfamily $(3,4)$. CST1 can inhibit the proteolytic activities of cysteine proteases and thus regulate tumor progression. Several studies have been conducted to confirm the role of CST1 or CST1 family members in tumor progression, for example, 
Inhibition of CST1 expression inhibits carcinogenesis and stem cell properties of papillary thyroid cancer, The mTOR/HIF1a/a-Enolase axis promotes thyroid cancer progression by regulating the expression of $\operatorname{CST} 1(5,6)$. Constructed an ECM-related signature in esophageal squamous cell carcinoma found that CST1 could be an independent prognostic factor and a potential biomarker of chemotherapy response in patients with Oesophageal Squamous Cell Carcinoma(7). Elevated expression levels of CST1 in breast cancer are closely associated with the progression and prognosis of breast cancer(8). A study confirmed that CST1 promotes invasion of gastric cancer cells through activation of Wnt Pathway(9). Upregulation of CST1 promotes hepatocellular carcinoma progression and predicts a poor prognosis(10). Let-7d inhibited colorectal carcinogenesis through the CST1/p65 pathway(11).

However, the expression level of CST1 in gastric cancer, the changes of the gene and whether it can be used as a prognostic molecular marker in gastric cancer have not been reported yet. We used bioinformatics analysis to elucidate the significance of CST1 in gastric cancer at several molecular levels.

\section{Materials And Methods}

\section{Oncomine database extracts data}

Oncomine database(https://www.oncomine.org/) is a platform based on a gene chip database and integrated data mining. In this database, screening and mining data conditions can be set according to their own needs. Our screening criteria are as follows: 1) "cancer type:gastric cancer"; 2) "gene:CST1"; 3) "Data Type:mRNA"; 4) "Analysis Type:Cancer vs Normal Analisis"; 5) the setting condition of the critical value is: ( $P$ value $<1 \mathrm{E}-4$, fold change $>2$, and gene rank=top $10 \%)$.

\section{Ualcan and TIMER database Analysis}

Ualcan(http://ualcan.path.uab.edu/index.html) is an online website for analyzing TCGA data, which can detect gene expression based on different factors in various cancers and normal tissues, such as grade, tumor stage, age, gender, race, and other pathological features. TIMER(http://timer.cistrome.org/) allows users to study the differential expression between tumor and adjacent normal tissues for any gene of interest across all TCGA tumors. We used the ualcan and TIMER database to detect the difference in CST1 expression in different types of gastric cancer.

\section{Selection of gastric cancer samples in TCGA}

Download mRNA expression information and clinical information of gastric cancer patients from TCGA database (https://cancergenome.nih.gov/). The main analysis was the difference in expression of cancer and paracancer CST1 in gastric cancer patients containing complete clinical information. A total of 354 GC patients and 30 normal controls were finally enrolled in the study.

\section{GSEA Analysis of the Hub Genes}


We divided GC samples into high-expression and low-expression groups based on the median expression levels of CST1. The effects of the expression of CST1 on multiple gene sets were analyzed in the relevant KEGG pathways using the molecular signatures database (MSigDB) (c2. cp.kegg.all.v7.1. symbols.gmt). The permutation of each analysis was 1,000 times. FDR q-value $<0.25$,NOM p-value $<0.05$, and |Normalized enrichment score (NES)|> 1 were used to identify significant differences.

\section{Kaplan-Meier Plotter Database Analysis}

The prognostic value of CST1,CST2 and CST4 expression including the overall survival (OS), first progression survival (FP), disease-free survival (DFS), and post progression surviaval (PPS) was evaluated in Kaplan-Meier Plotter(http://kmplot.com/analysis/).

\section{GeneMANIA Database Analysis}

We analyzed the genes that interacted most significantly with CST1 on GeneMANIA(http://genemania.org/), following the website database guidelines.

\section{cBioPortal database Analysis}

A total of 1460 patients with GC was obtained from cBioPortal database(https://www.cbioportal.org/) to analyze the genetic alteration of CST1. The mutation frequency and sites of CST1 in GC can be found in the database.

\section{Antibodies and reagents}

CST1(16025-1-AP) was purchased from Proteintech. $\beta$-actin(A01010) was purchased from Abbkine. The small interfering RNA constructs against CST1 used in this study was from GenePharma.CCK-8 and $0.01 \%$ Crystal Violet Staining Solution was purchased from Servicebio. PMSF was purchased from Beyotime Biotechnology.

\section{Western blot}

Cells cultured were harvested in 200ul RIPA buffer (Beyotime Biotechnology), The supernatant was obtained by centrifugation at $13000 \mathrm{rpm}, 10 \mathrm{~min}$, and the protein was cooked at $96^{\circ} \mathrm{C}$ for $10 \mathrm{~min}$. Protein samples were boiled and separated on 10\% SDS-PAGE gels followed by transferring to PVDF membranes (Millipore, Billerica, MA, USA). After blocking with 5\% nonfat milk in TBST for 30 min, the membranes were incubated with specific primary antibodies overnight at $4{ }^{\circ} \mathrm{C}$. Finally, antibody-bound proteins were detected by chemiluminescence (BIORAD, USA).

\section{Cell culture}

NCI-N87 and AGS cells purchased from China Center for Type Culture Collection(CCTCC). MGC803 cells and SGC7901 cells were obtained from the Laboratory of Medical Translation Platform, Academy of Medical Sciences, Zhengzhou University.The cells were grown in 1640 and F12K, supplemented with 10\% 
fetal bovine serum (FBS) (Biological Industries) and $0.5 \%$ antibiotics, at $37^{\circ} \mathrm{C}$ with $5 \% \mathrm{CO} 2$ incubator. The 1640 was purchased from Servicebio(wuhan). The F12K was purchased from Gbico.

\section{Cell proliferation assay}

CCK-8 assay analyzes the effect of silencing CST1 on the proliferative capacity of gastric cancer cells. NCI-N87 cells and AGS cells were seeded into 96-well plates at a density of 5×103 cells/well, and incubated for 24 hours, 48 hours and 72 hours, respectively. Add CCK-8 reagent at a ratio of 1:10 each well. Absorbance was measured at a wavelength of $450 \mathrm{~nm}$ using a microplate reader (Bio-Rad Laboratories, Inc, Hercules, CA, USA). Each experiment was performed in triplicate.

\section{Colony-formation assay}

NCl-N87 cells and AGS cells $(8 \times 103 /$ well) in a 6 -well plate cultured in $1 \mathrm{ml}$ of $0.33 \%$ BME (Eagle basal medium, Sigma-Aldrich Corp.) agar (Sigma-Aldrich Corp.) The cells were maintained in a $37^{\circ} \mathrm{C}, 5 \% \mathrm{CO} 2$ incubator for 7-10 days,their colonies were stained with Nitrotetrazolium Blue chloride (NBT) overnight at $37^{\circ} \mathrm{C}$ then were observed by microscopy, and statistical analysis was performed.

\section{Transwell assay}

Cell-invasion and migration ability was evaluated using the transwell assay. Next, $200 \mu \mathrm{L}$ of serum-free 1640 and $\mathrm{F} 12 \mathrm{~K}$ medium at a density of $5 \times 10^{5} \mathrm{cell} / \mathrm{mL}$ suspension was added into the upper layer of the transwell chamber (Corning) covered with Matrigel (BD Biosciences, San Jose, CA, USA), while $600 \mu \mathrm{L}$ of $20 \%$ FBS medium was added to the lower chamber. The transwell chamber was cultured for $48 \mathrm{~h}$. Migration assay with $5 \times 10^{4} \mathrm{NCI}-\mathrm{N} 87$ cells and AGS cells. after $12 \mathrm{~h}$, the cells were fixed with $4 \%$ Paraformaldehyde for $30 \mathrm{~min}$. Next, cells were stained with $0.01 \%$ crystal violet solution for $30 \mathrm{~min}$, PBS wash 3 times, 5 min each time. Wipe off the upper layer of cells with a cotton swab. The invaded cells were observed and images taken under an inverted microscope (Leica), and statistical analysis was performed.

\section{Statistical analysis}

The expression of CST1 and other clinicopathological features were analyzed by student's, $t$ test, $\chi 2$ test with Spss21.0 software. Kaplan-Meier method and log-rank, t-test were used to make OS curves.

\section{Result}

\section{Expression Analysis of CST1}

CST1 are expressed in a wide range of cancers according to The Cancer Genome Atlas (TCGA) UALCAN database. CST1 expression is lowest in the Brain lower grade glioma (LGG) dataset (median value 0) and highest in the Pancreatic adenocarcinoma (PAAD) dataset (median value 7.181), CST1 expression in gastric cancer(GC) is second only to PAAD(Fig1A).compared with normal tissues, most tumor types show 
significantly higher expression of CST1,and is most evident in GC(Figure1B). Next, we used oncomine database data to analyze the expression level of CST1 in cancer. The results showed that there were 388 studies involving in cancer, 38 of them were statistically different, and 37 of them had increased expression of CST1 in tumors(Figure2A). The results of 4 studies on gastric cancer suggested that the expression of CST1 in cancer tissues was significantly higher than that in normal tissues. There was significant difference in Cho gastric (Fig2B,p<0.0001),Cui gastric(Figure2C, $p<0.0001$ ), Derrico gastric (Fig2D,p<0.005), and Wang gastric (Fig2E,p < 0.0001).

\section{The expression of CST1 in different types of gastric cancer}

We used a ualcan online database to detect the expression level of CST1 in the classification of different influencing factors of gastric cancer. The results suggested that the expression of CST1 in primary tumor vs normal (Figure3A), age vs normal (Figure3B), gender vs normal (Figure3C), grade vs normal (Fig3D), stage vs normal (Figure3E), histological subtypes vs normal(Fig3F) was higher than that in normal tissues $(p<0.05)$.

\section{Promoter methylation levels of CST1 in different types of gastric cancer}

Next, We used a ualcan online database to detect the promoter methylation levels of CST1 in the classification of different influencing factors of gastric cancer. The results suggested that the promoter methylation levels of CST1 in primary tumor vs normal (Figure4A), gender vs normal (Figure4B), age vs normal (Figure4C), grade vs normal (Figure4D), TNM vs normal (Figure4E), stage vs normal (Figure4F) was lower than that in normal tissues $(p<0.05)$.

\section{Correlation Between the Expression of CST1 and Prognosis}

We employed the KM plotter database to explore the effect of CST1 expression on the survival of patients of GC, we detected a significant correlation between prognosis and CST1 expression (Figure5). We revealed that a higher CST1 expression level was significantly related to a poorer prognosis in GC (OS, HR $=1.7,95 \% \mathrm{Cl}=1.42-2.02, \mathrm{p}=2.9 \mathrm{e}-09$; First progression survival $(\mathrm{FPS}), \mathrm{HR}=1.63,95 \% \mathrm{Cl}=1.32-2.01, \mathrm{p}=$ 3.9e-06; post progression survival (PPS), $\mathrm{HR}=1.92,95 \% \mathrm{Cl}=1.51-2.43, \mathrm{p}=4.4 \mathrm{e}-08$;) (Figures5A, Figure5B, Figure5C).

\section{CST1 expression and prognosis in TCGA GC dataset}

The data of 354 patients with gastric cancer expressing CST1 were downloaded from the TCGA database, and the expression of CST1 in patients with gastric cancer was analyzed. CST1 expression level was most significantly upregulated (Fold-change $=20.94, p<0.001)$ ( Figure6A, Figure6B). The patients were divided into two groups according to the median expression of CST1 (35.107): high expression of CST1 $(n=177)$ and low expression of FBXO22 $(n=177)$. The clinicopathological features of patients with gastric cancer were shown in Table1. The results showed that the expression of CST1 was positively correlated with the OS(Figure6C). 


\section{Functional Annotations and Predicted Signaling Pathways}

To identify the CST1-related pathways activated in GC, we conducted GSEA between low and high CST1 expression datasets. Significant differences (false-discovery rate $<0.25, p<0.05$ ) in the enrichment of the Molecular Signature database Collection are shown in Figure 7. The results showed that CST1 was mainly involved in Coagulation(Figure7A) and Epithelial-Mesenchymal-Transition(Figure7B), Complement(Figure7C), Apoptosis(Figure7D), Glycolysis(Figure7E). These results showed that high expression of CST1 was closely associated with malignancy in GC.

\section{Enrichment Analysis of CST1-Related Genes and CST1,CST2 and CST4 Genetic Alterations in Patients with GC}

We investigated the interaction network for CST1 by the GeneMANIA database. Figure 8A shows the top 20 genes and their relationship with CST1. Among them, CST2 and CST4 of the same family of CST1 are extremely correlated with it. Next, we investigated the frequency of alterations CST1,CST2 and CST4 in five groups of GC cases via the cBioPortal web resource(Figure8A). Overall, 1460 samples from TCGA GC database were studied (Figure8B,Figure8C), and the mutation rate was found to be low $(1.36-3.16 \%)$ in Gastric Cancer (OncoSG, 2018),Stomach Adenocarcinoma (TCGA, Nature 2014),Stomach Adenocarcinoma (TCGA, Firehose Legacy),Stomach Adenocarcinoma (TCGA, PanCancer Atlas),Stomach Adenocarcinoma (Pfizer and UHK, Nat Genet 2014). The type as well as the location of distinct mutations are indicated in Figure8D.

\section{KM plotter database analysis of the relationship between CST2 and CST4 expression and prognosis in gastric cancer}

We employed the KM plotter database to explore the effect of CST2 and CST4 expression on the survival of patients of GC, we detected a significant correlation between prognosis and CST1 expression (Figure9). We revealed that a higher CST2 expression level was significantly related to a poorer prognosis in $\mathrm{GC}(\mathrm{OS}, \mathrm{HR}=1.82,95 \% \mathrm{Cl}=1.53-2.18, \mathrm{p}=1.8 \mathrm{e}-11$; First progression survival $(\mathrm{FPS}), \mathrm{HR}=1.84,95 \% \mathrm{Cl}=$ 1.5-2.25, $\mathrm{p}=2.7 \mathrm{e}-09$; post progression survival (PPS), $\mathrm{HR}=2.07,95 \% \mathrm{Cl}=1.65-2.61, \mathrm{p}=2.2 \mathrm{e}-10$;) (Figures9A, Figure9B, Figure9C). Higher CST4 expression level was significantly related to a poorer prognosis in $\mathrm{GC}(\mathrm{OS}, \mathrm{HR}=1.52,95 \% \mathrm{Cl}=1.28-1.8, \mathrm{p}=1.5 \mathrm{e}-06$; First progression survival $(\mathrm{FPS}), \mathrm{HR}=1.54$, $95 \% \mathrm{Cl}=1.24-1.9, \mathrm{p}=5.9 \mathrm{e}-05$; post progression survival $(\mathrm{PPS}), \mathrm{HR}=1.56,95 \% \mathrm{Cl}=1.23-1.99, \mathrm{p}=$ 0.00025;) (Figure9D, Figure9E, Figure9F).

\section{Knockdown of CST1 expression inhibited the proliferation, colony formation, migration and invasion ability of gastric cancer cells.}

We first examined the protein expression level of CST1 in four gastric cancer cell lines and found that CST1 was expressed at a higher level in AGS and NCI-N87 than in MGC803 and SGC7901 (Figure10A). Secondly, we knocked down the expression of CST1 in AGS and NCI-N87 cells (Figure10B). Anchorageindependent colony-formation assay showed that knockdown of CST1 significantly inhibited the 
clonogenic ability of AGS and NCI-N87 cells (Figure10C, Figure10D). We then used Transwell assay to detect the effect of CST1 on the migration and invasion ability of gastric cancer cells, and found that inhibition of CST1 expression significantly inhibited the migration and invasion ability of AGS and NClN87 cells (Figure11A, Figure11B).

\section{Discussion}

Gastric cancer is one of the most malignant cancers worldwide and a large number of patients lose their lives every year. The reason for the high mortality rate of gastric cancer is the lack of early markers.

The molecular role of CST1 in several cancers has been elucidated, for example,CST1 acts as an oncogene in ER + breast cancer, and CST1 contributes to cancer development by regulating the $\mathrm{ERa} / \mathrm{PI}$ IK/AKT/ERa loopback pathway in ER + breast cancer(12).CST1 was confirmed as a prognostic marker for cholangiocarcinoma by weighted gene correlation network analysis(13, 14). CST1 family members can regulate metastasis in triple-negative breast cancer(15). We analyzed the differential genes by downloading TCGA GC data and found that CST1 was most significantly upregulated in gastric cancer. We analyzed the relationship between CST1 and gastric cancer prognosis and genetic alterations by Oncomine,Ualcan ,Kaplan-Meier Plotter and cBioPortal database, and clarified the role of CST1 as a prognostic biomarker in gastric cancer, gastric cancer patients with high CST1 expression have poor prognosis. This is consistent with previous studies that confirmed the role of CST1 as a proto-oncogenic molecule in gastric cancer(9). In contrast, our study focused on confirming the role of CST1 in gastric cancer through TCGA cancer database and analyzed the expression and gene mutation of CST1 in gastric cancer.CST1 is an independent prognostic factor for non-small cell lung cancer (NSCLC) patients(16).CST1 is a prognostic marker and therapeutic target for colorectal cancer patients(17). In vitro functional assay confirmed that inhibition of CST1 significantly inhibited the proliferation, migration and invasion ability of gastric cancer cells. The above study illustrates that CST1 acts as a protooncogenic factor in several cancers, and through cancer database analysis we confirmed that CST1 is closely associated with poor prognosis in gastric cancer patients and can be used as a prognostic marker in gastric cancer, the only drawback is that we did not do molecular biology experiments to confirm the role of CST1 in gastric cancer.

\section{Conclusions}

In conclusion, CST1 was highly expressed in gastric cancer and patients with high CST1 expression had a poorer prognosis, and CST1 was found to promote the proliferation and metastatic ability of gastric cancer cells. Our data confirm the prognostic value of CST1 as a biomarker for gastric cancer.

\section{Abbreviations}

GC Gastric cancer 
EMT Epithelial-mesenchymal transition

\section{Declarations}

Ethics approval and consent to participate

Not applicable.

Consent for publication

Not applicable.

Availability of data and materials

Raw data of GC cohort was acquired from TCGA portal (https://portal.gdc.

cancer.gov/). Analyzed data is available within the materials and results.

Competing interests

All authors declare there are no any competing interests with regard to the publication of the study.

Funding

No.

Authors' Contributions:

XTT and FWJ collected data,performed data analysis and wrote the manuscript. XTT, FWJ, LHY, JST, GXX and DYW cultured cells and performed functional experiments,FWJ and YBZ performed statistical analysis. XTT contributed to data interpretation, and manuscript revision.

Acknowledgments

Not applicable.

\section{Contributor Information}

TingTing Xu,E-mail: xutingtingp@126.com

\section{References}

1. Bray F, Ferlay J, Soerjomataram I, Siegel RL, Torre LA and Jemal A: Global cancer statistics 2018: GLOBOCAN estimates of incidence and mortality worldwide for 36 cancers in 185 countries. CA Cancer J Clin 68: 394-424, 2018. 
2. Chen W, Zheng R, Baade PD, et al.: Cancer statistics in China, 2015. CA Cancer J Clin 66: 115-132, 2016.

3. Barrett AJ: The cystatins: a diverse superfamily of cysteine peptidase inhibitors. Biomed Biochim Acta 45: 1363-1374, 1986.

4. Saitoh E, Kim HS, Smithies $\mathrm{O}$ and Maeda N: Human cysteine-proteinase inhibitors: nucleotide sequence analysis of three members of the cystatin gene family. Gene 61: 329-338, 1987.

5. Ding J, Wang X, Gao J and Song T: Silencing of cystatin SN abrogates cancer progression and stem cell properties in papillary thyroid carcinoma. FEBS Open Bio2021.

6. Liu Y, Liao L, An C, et al.: alpha-Enolase Lies Downstream of mTOR/HIF1alpha and Promotes Thyroid Carcinoma Progression by Regulating CST1. Front Cell Dev Biol 9: 670019, 2021.

7. Zhang H, Shi Q, Yang Z, et al.: An Extracellular Matrix-Based Signature Associated With Immune Microenvironment Predicts the Prognosis and Therapeutic Responses of Patients With Oesophageal Squamous Cell Carcinoma. Front Mol Biosci 8: 598427, 2021.

8. Dai DN, Li Y, Chen B, et al.: Elevated expression of CST1 promotes breast cancer progression and predicts a poor prognosis. J Mol Med (Berl) 95: 873-886, 2017.

9. Chen S, Liu Y, Zhang K and Chen L: CST1 Promoted Gastric Cancer Migration and Invasion Through Activating Wnt Pathway. Cancer Manag Res 13: 1901-1907, 2021.

10. Cui Y, Sun D, Song R, et al.: Upregulation of cystatin SN promotes hepatocellular carcinoma progression and predicts a poor prognosis. J Cell Physiol 234: 22623-22634, 2019.

11. Jiang J, Liu HL, Tao L, et al.: Let7d inhibits colorectal cancer cell proliferation through the CST1/p65 pathway. Int J Oncol 53: 781-790, 2018.

12. Liu Y, Ma H, Wang Y, Du X and Yao J: Cystatin SN Affects Cell Proliferation by Regulating the ERalpha/PI3K/AKT/ERalpha Loopback Pathway in Breast Cancer. Onco Targets Ther 12: 1135911369, 2019.

13. Liu J, Liu W, Li H, et al.: Identification of key genes and pathways associated with cholangiocarcinoma development based on weighted gene correlation network analysis. PeerJ 7: e7968, 2019.

14. Tian A, Pu K, Li B, et al.: Weighted gene coexpression network analysis reveals hub genes involved in cholangiocarcinoma progression and prognosis. Hepatol Res 49: 1195-1206, 2019.

15. Johnstone CN, Pattison AD, Gorringe KL, et al.: Functional and genomic characterisation of a xenograft model system for the study of metastasis in triple-negative breast cancer. Dis Model Mech 112018.

16. Cao X, Li Y, Luo RZ, et al.: Expression of Cystatin SN significantly correlates with recurrence, metastasis, and survival duration in surgically resected non-small cell lung cancer patients. Sci Rep 5: 8230, 2015.

17. Li T, Xiong Q, Zou Z, Lei X, Jiang Q and Liu D: Prognostic significance of cystatin SN associated nomograms in patients with colorectal cancer. Oncotarget 8: 115153-115163, 2017. 


\section{Tables}

Table1. Patient characteristics and associations with CST1 expression in TCGA data 


\begin{tabular}{|c|c|c|c|c|}
\hline \multirow[t]{2}{*}{ Clinical characteristics } & \multicolumn{2}{|c|}{ patients $₫ 354 \rrbracket$} & \multicolumn{2}{|l|}{ CST1 } \\
\hline & Number & $\%$ & High & low \\
\hline \multicolumn{5}{|l|}{ Age } \\
\hline$>60$ & 237 & 67 & 126 & 111 \\
\hline$\leq 60$ & 117 & 33 & 51 & 66 \\
\hline \multicolumn{5}{|l|}{ Gender } \\
\hline Male & 224 & 63 & 113 & 111 \\
\hline Female & 130 & 37 & 64 & 66 \\
\hline \multicolumn{5}{|l|}{ Stage } \\
\hline NA & 17 & 5 & 13 & 4 \\
\hline Stagel & 42 & 12 & 19 & 23 \\
\hline Stagell & 112 & 32 & 51 & 61 \\
\hline Stagelll & 147 & 42 & 77 & 70 \\
\hline StagelV & 36 & 9 & 18 & 18 \\
\hline \multicolumn{5}{|l|}{ TNM stage } \\
\hline \multicolumn{5}{|l|}{$\mathrm{T}$} \\
\hline TX & 8 & 2 & 7 & 1 \\
\hline T1 & 16 & 5 & 4 & 12 \\
\hline T2 & 75 & 21 & 36 & 39 \\
\hline T3 & 163 & 46 & 85 & 78 \\
\hline $\mathrm{T} 4$ & 92 & 26 & 45 & 47 \\
\hline \multicolumn{5}{|l|}{$\mathbf{N}$} \\
\hline NX & 16 & 5 & 11 & 5 \\
\hline NO & 105 & 30 & 44 & 61 \\
\hline N1 & 95 & 27 & 43 & 52 \\
\hline N2 & 64 & 18 & 35 & 29 \\
\hline N3 & 74 & 20 & 44 & 30 \\
\hline \multicolumn{5}{|l|}{ M } \\
\hline$M X$ & 13 & 4 & 7 & 6 \\
\hline MO & 317 & 90 & 159 & 158 \\
\hline
\end{tabular}

Page 12/19 


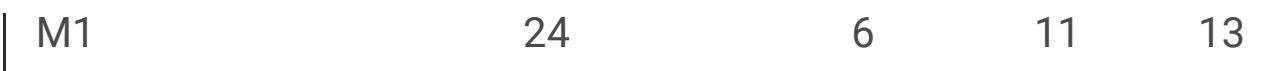

Figures

A

Expression of CST1 across TCGA tumors

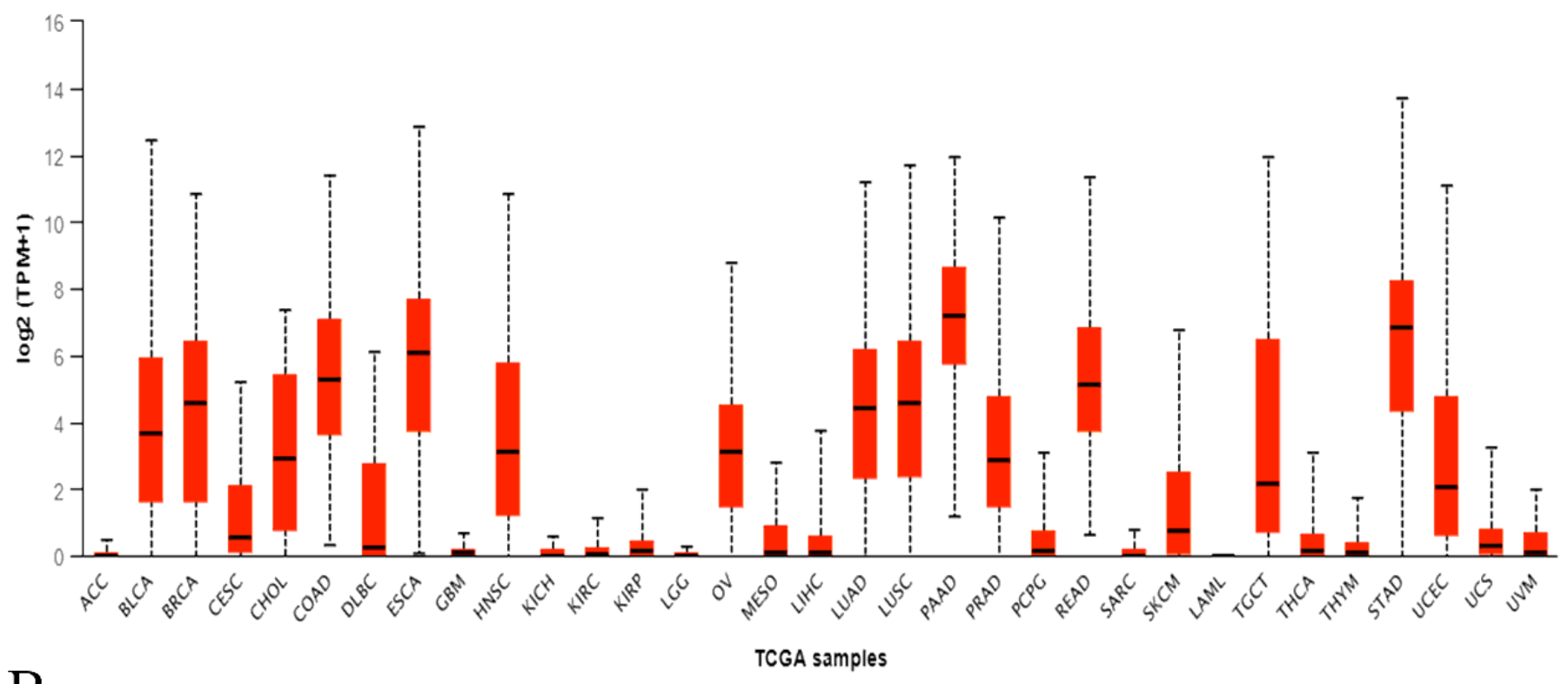

B

Expression of CST1 across TCGA cancers (with tumor and normal samples)

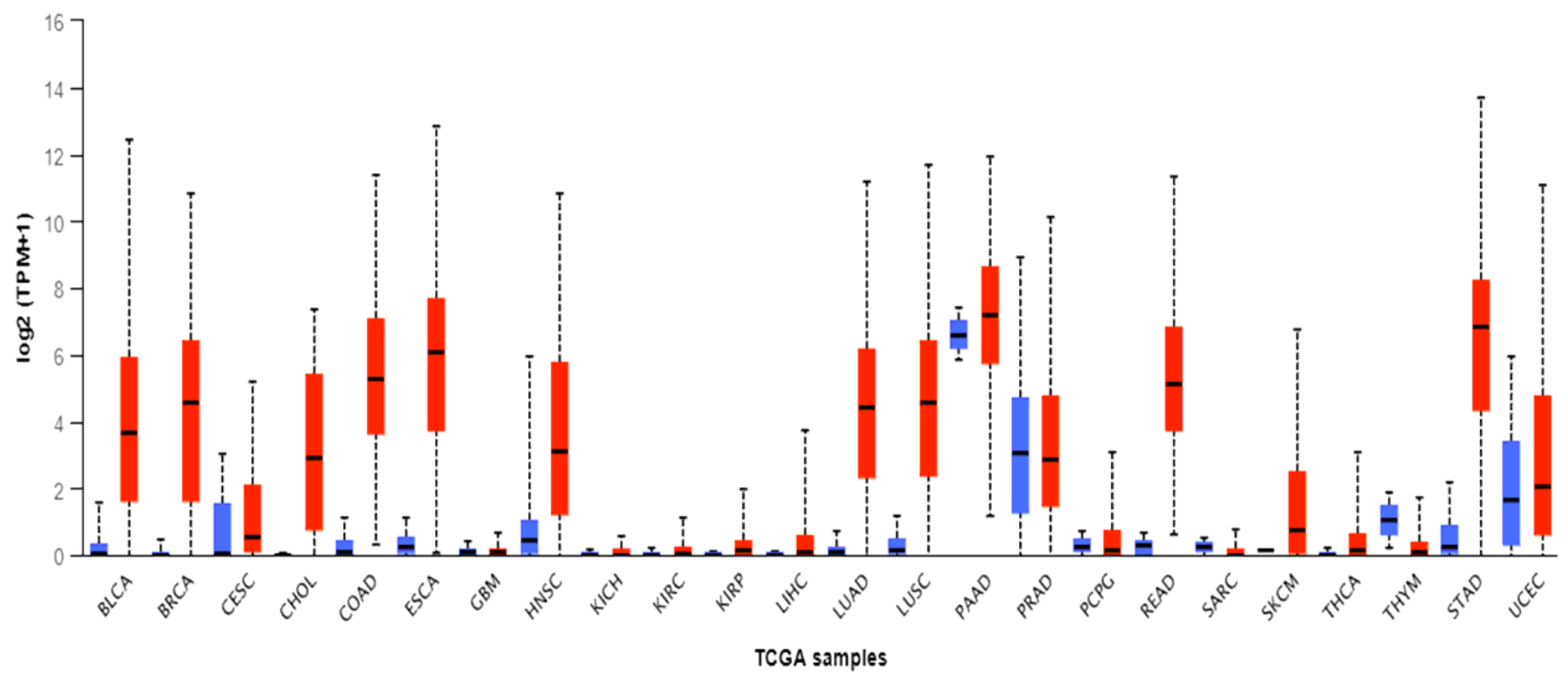

Figure 1 
Expression of CST1 in cancer. (A) Expression of CST1 among various cancer types. (B) Comparison of the expression of CST1 between tumor and normal tissues. The images and significance are from ULCAN database.

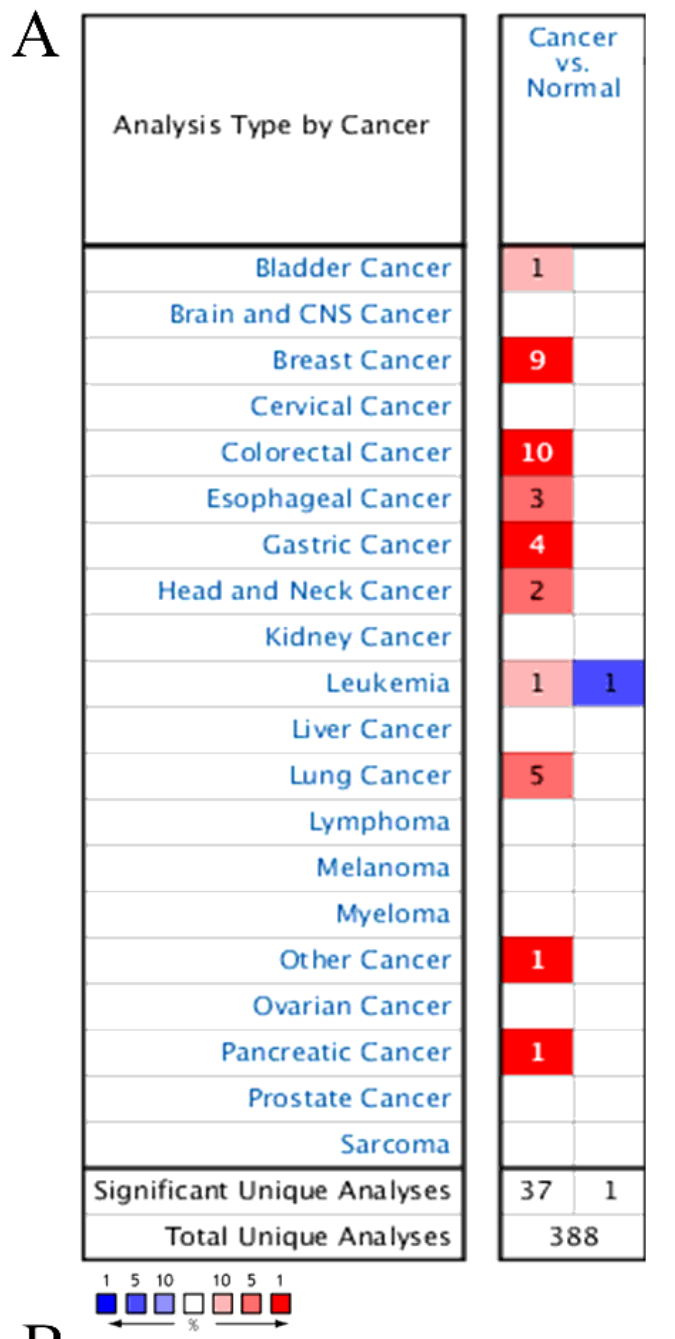

CST1 expression in Cho gastric

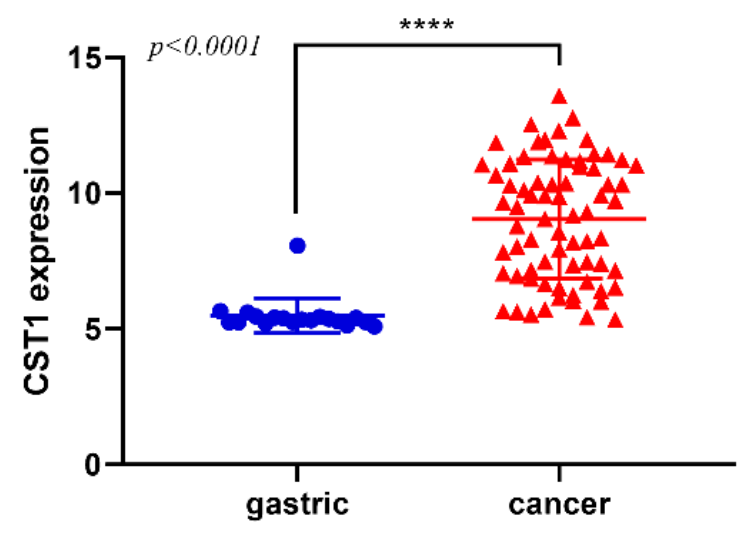

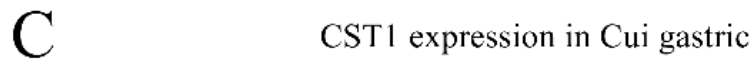

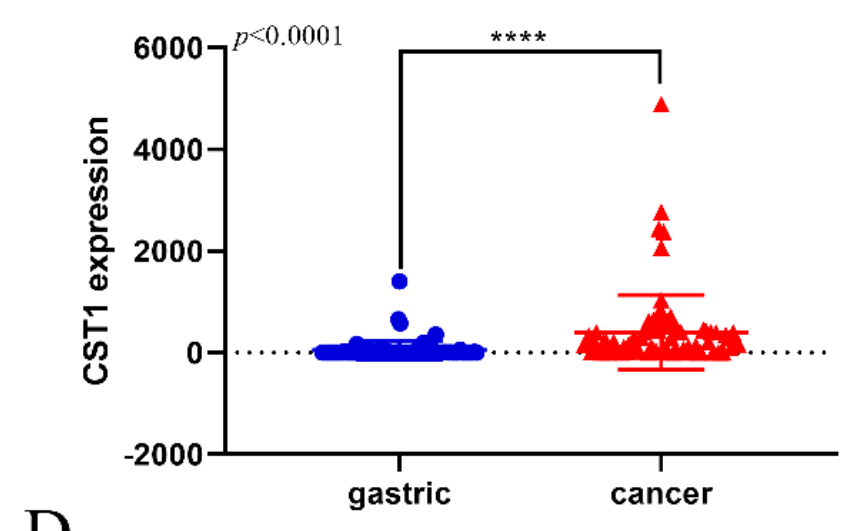

CST1 expression in DErrico gastric

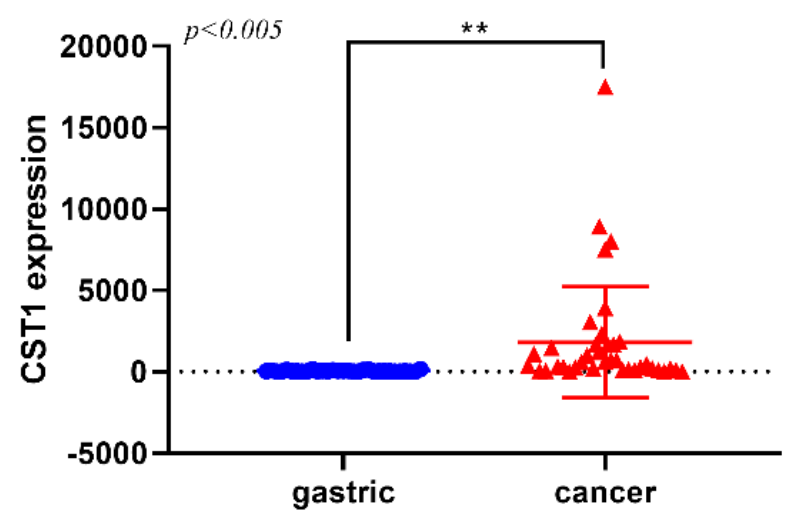

$\mathrm{E}$

CST 1 expression in Wang gastric

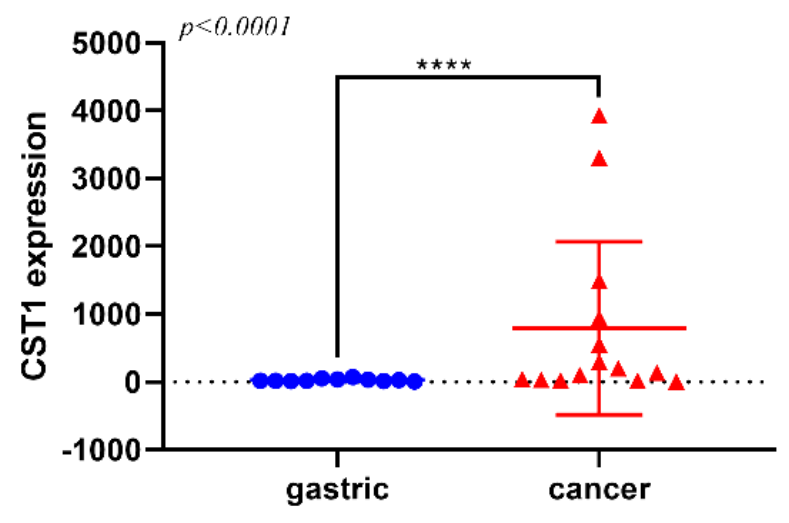

Figure 2 
Expression of CST1 in oncomine database. (A) The number of data sets presented in this graph belongs to the over-expression (red) or down-expression (blue) of target genes (cancer and normal tissues), which is of statistical significance. (B-E) The CST1 mRNA levels were significantly higher in GC than in Normal according to the Cui gastric Statistics, Derrico gastric Statistics, Cho gastric Statistics and Wang gastric Statistics datasets.

\section{Figure 3}

Box plots showing CST1 expression levels in GC via the UALCAN database analysis. (A) Box plots show CST1 protein expression levels in normal tissues vs.GC tissues (B) in normal tissues vs. GC tissues in different age. (C) in normal tissues vs. GC tissues in different gender. (D) in normal tissues vs. GC tissues in different grade. (E) in normal tissues vs. GC tissues in different stage. $(F)$ in normal tissues vs. GC tissues in different histological subtypes. 
A

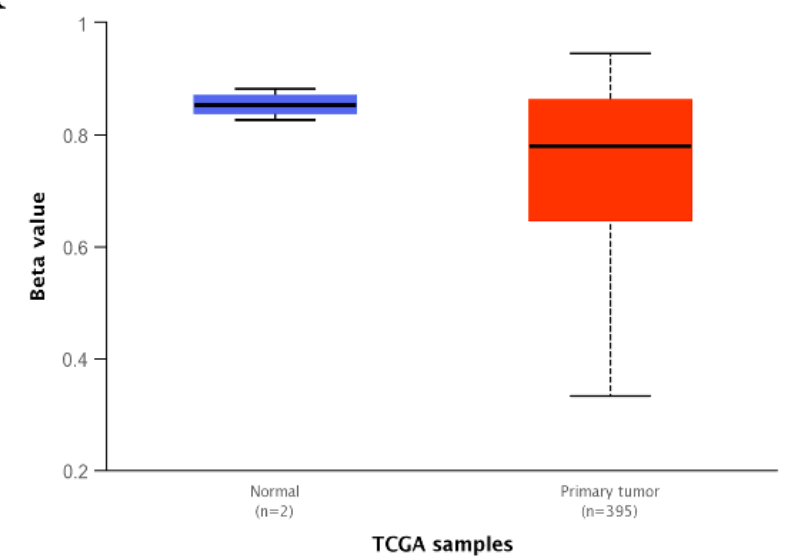

$\mathrm{C}$

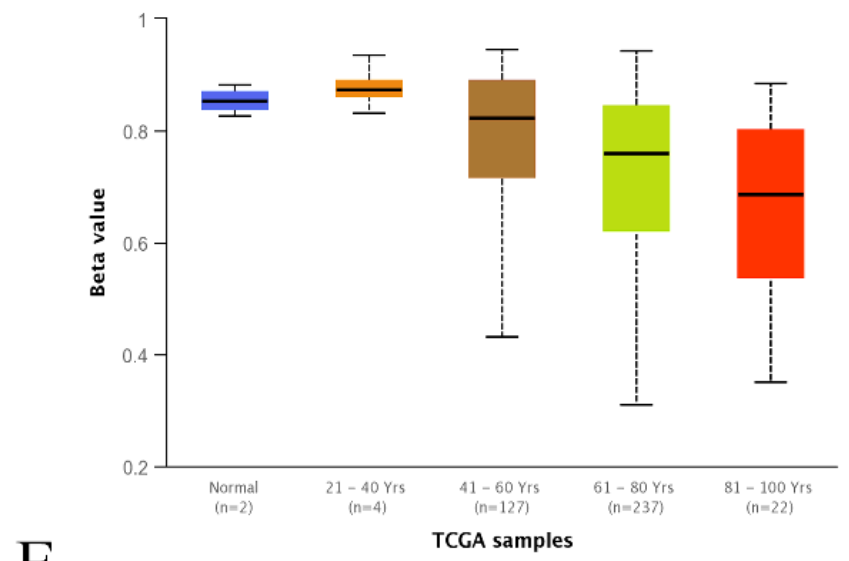

$\mathrm{E}$

Promoter methylation level of CST1 in STAD

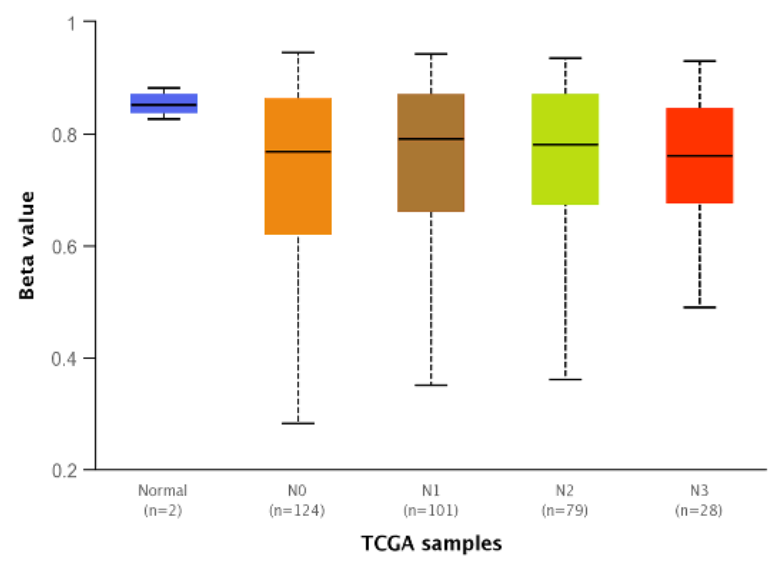

$\mathrm{B}$

Promoter methylation level of CST1 in STAD

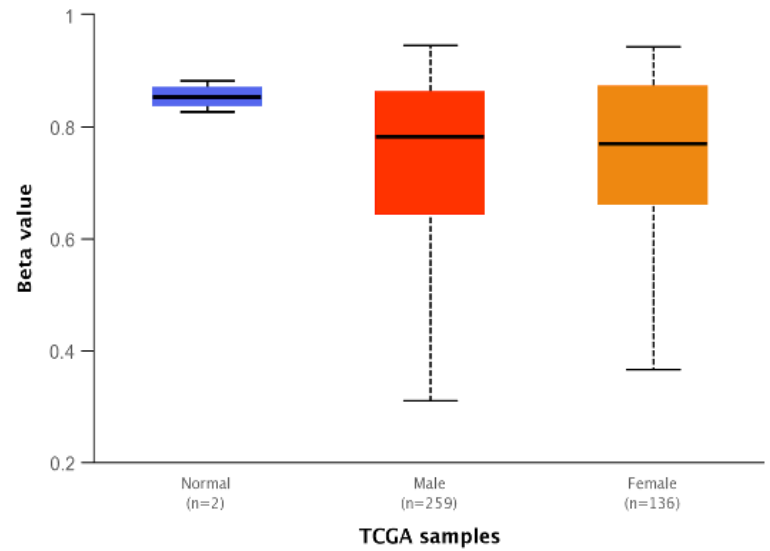

$\mathrm{D}$

Promoter methylation level of CST1 in STAD

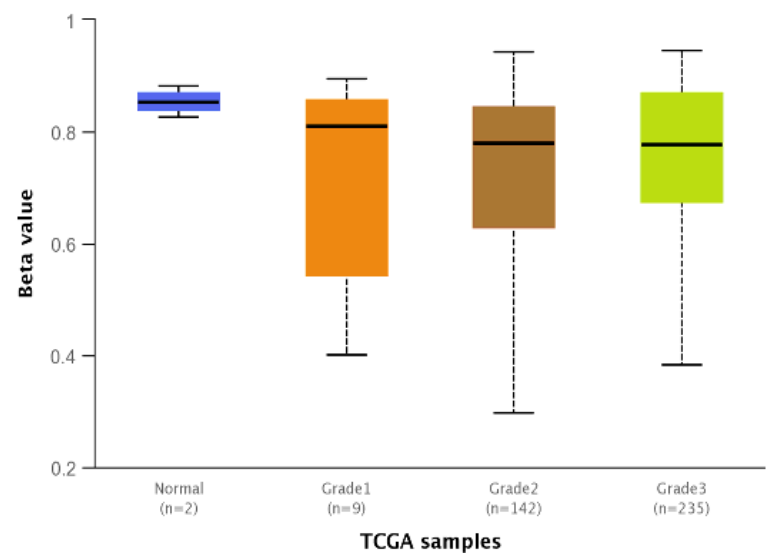

$\mathrm{F}$

Promoter methylation level of CST1 in STAD

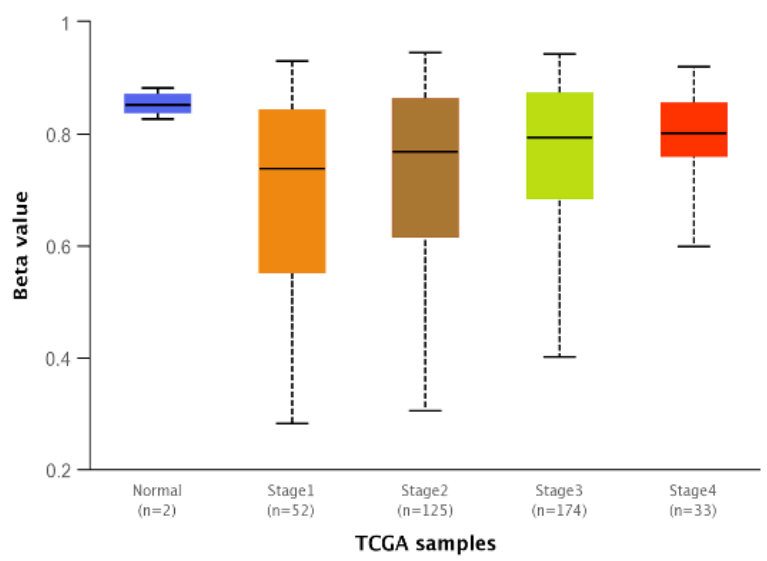

\section{Figure 4}

Box plots showing CST1 promoter methylation levels in GC via the UALCAN database analysis. (A) Box plots show CST1 protein expression levels in normal tissues vs.GC tissues (B) in normal tissues vs. GC tissues in different gender. (C) in normal tissues vs. GC tissues in different age. (D) in normal tissues vs. GC tissues in different grade. (E) in normal tissues vs. GC tissues in different TNM. (F) in normal tissues vs. GC tissues in different stage. 


\section{Figure 5}

CST1 expressions were closely related to the prognosis of GC patients. (A) Overall survival rate was assessed in TCGA GC patients. (B) First progression survival rate was analyzed in TCGA GC patients. (C) Post progression survival rate was analyzed in TCGA GC patients. Abnormal expression and low expression were identified with the auto best cutoff.

\section{Figure 6}

CST1 expression and prognosis in the TCGA GC dataset. (A) Heat map of differential genes in TCGA GC dataset. (B) Expression levels of CST1 in GC tissue vs. normal tissue. (C) Survival differences between high and low CST1 expression in GC patients 

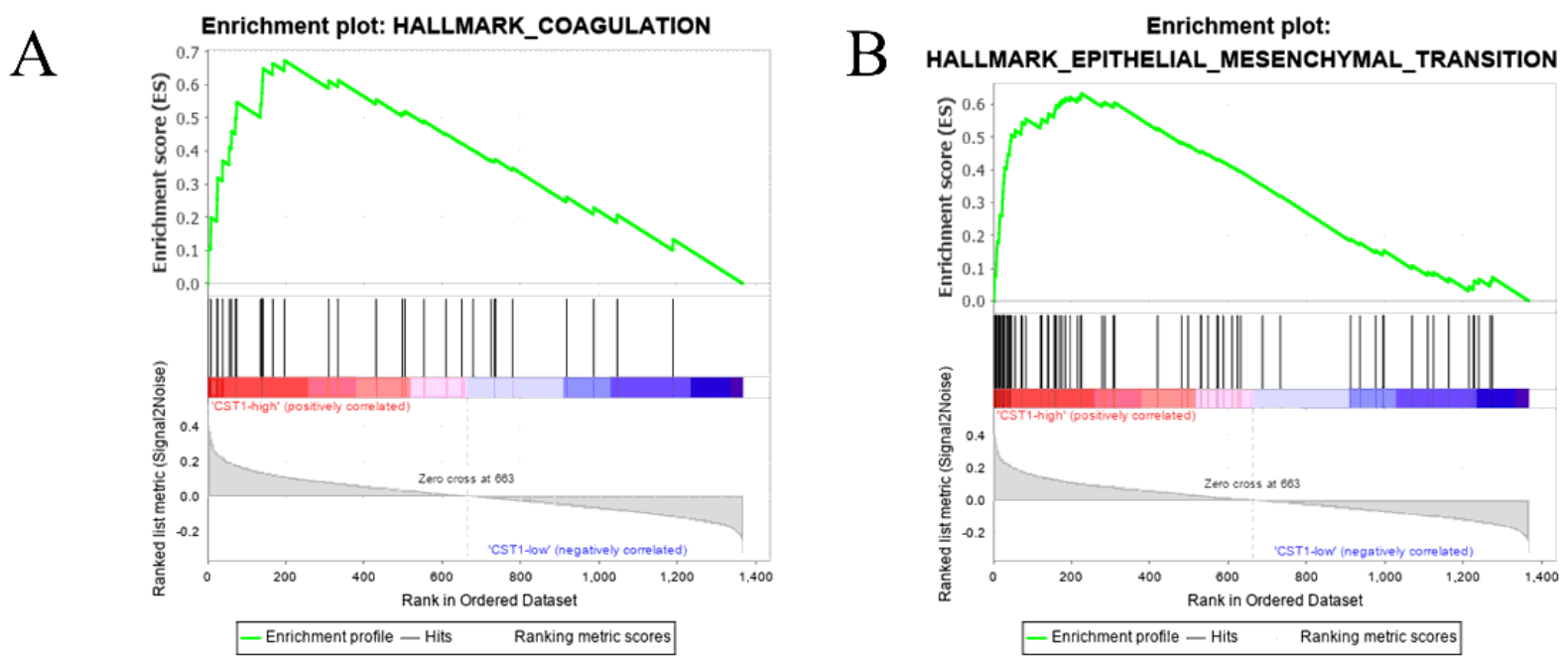

$\mathrm{C}$

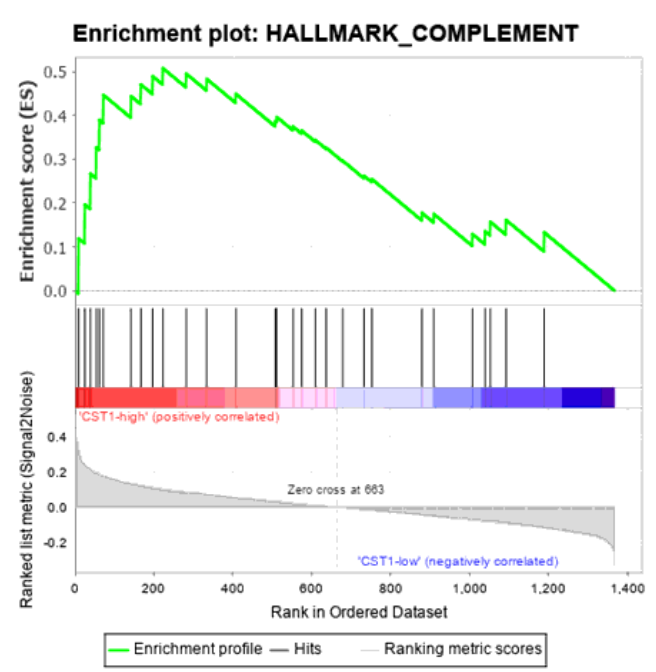

$\mathrm{D}$

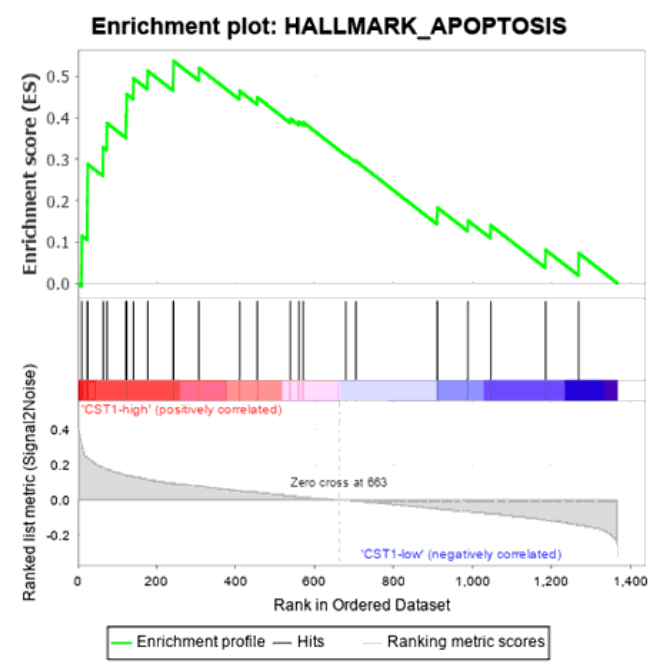

$\mathrm{E}$

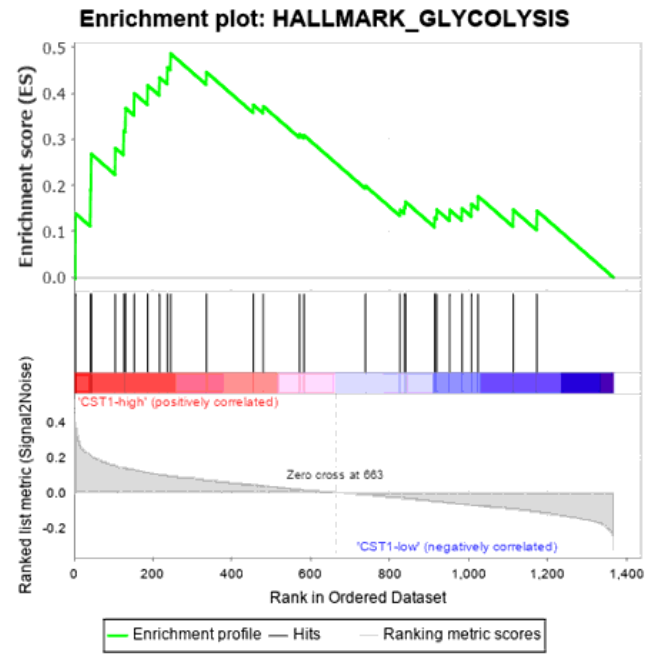

\section{Figure 7}

The Gene Set Enrichment Analysis (GSEA) of the relationship between the expression level of CST1 in the TCGA GC dataset. The most involved significant hallmark pathways which were closely correlated with CST1 in GC obtained by GSEA. (A) Coagulation. (B) Epithelial-Mesenchymal-Transition. (C) Complement. (D) Apoptosis. (E) Glycolysis. NES: normalized enrichment score; FDR: false discovery rate. 


\section{Figure 8}

Enrichment Analysis of CST1-Related Genes and CST1,CST2 and CST4 Genetic Alterations in Patients with GC (A) Using the GeneMANIA approach, top 20 CST1-correlated genes were analyzed. (B) Alteration frequency in GC. (C) Mutation types of CST1,CST2 and CST4. (D) Mutation sites of CST1.

\section{Figure 9}

CST2 and CST4 expressions were closely related to the prognosis of GC patients. (A) Overall survival rate of CST2 was assessed in TCGA GC patients. (B) First progression survival rate of CST2 was analyzed in TCGA GC patients. (C) Post progression survival rate of CST2 was analyzed in TCGA GC patients. (D) Overall survival rate of CST4 was assessed in TCGA GC patients. (E) First progression survival rate of CST4 was analyzed in TCGA GC patients. (F) Post progression survival rate of CST4 was analyzed in TCGA GC patients. Abnormal expression and low expression were identified with the auto best cutoff.

\section{Figure 10}

CST1 promotes the proliferation ability of gastric cancer cells.(A) Protein levels of CST1 in four gastric cancer cell lines.(B) Knockdown of CST1 expression in AGS and NCI-N87 cells.(C) Cell proliferation assay of AGS and NCl-N87 after knockdown of CST1.(D) Colony formation proliferation assays of AGS and NClN87 after knockdown of CST1.

\section{Figure 11}

CST1 promotes the migration and invasive ability of gastric cancer cells.

(A) Cell migration assays of AGS and NCI-N87 after knockdown of CST1.(B) Cell invasion assays of AGS and NCI-N87 after knockdown of CST1. 